\title{
Paráme tros antropométricos en varones adolescentes chilenos de nivel socioeconómico medio-alto
}

\author{
Dr. Francisco Beas F, 1; Dr. Radek Barrera R.1,2; Dr. Luis Corvalán M.2; \\ Dra. Amanda Cortínez R. ${ }^{\text {; }}$ Dra. Martha Eggers M. ${ }^{\text {; }}$ Dra. Cecilia Herriquez del V.l \\ Selected anthropometric aspects in male chilean adolescents
}

Some anthropometric characteristics in male Chilean adolescents, of mid-high socioeconomic level were registered as part of a larger research dealing with the clinical, psychological and cultural characteristics of Chilean adolescents. Observed values for height, weight and gowth spurt time were similar to those described for British adolescents (Tanner) and are significantly higher than those observed in the Chilean adolescents from a lower socioeconomical stratum. It is postulated that height is more influenced by socioeconomical conditions than by genetic factors. Trunk and extremities grow in the same proportions during adolescence. The growth of the thrunk coincides with the growth's spurt at 14 years of age. The extremities grow in two peaks, the first at 10 years and the second at 14 years.

(Key words: Body, growth, adolescent, males).

Los cambios biológicos son un fenómeno tan evidente en la adolescencia que incluso se han usado como indicadores del inicio de esta etapa. En nuestro pais no contamos con muchos estudios acerca del patrón característico del desarrolio biológico del adolescente y tampoco disponemos de estudios empíricos acerca de su interrelación con el desarrollo psicológico. Pensamos que para lograr resolver estos problemas es indispensable un trabajo interdisciplinario usando

1. Instituto de Investigaciones Clínicas, Hospital Paula Jaraquemada. División Ciencias Médicas Sur, Facultad de Medicina, Universidad de Chile.

2. Servicio de Endpcrinología, Departamento de Medicina Interna, Hospital Paula Jaraquemada. instrumentos de medición que den cuenta en forma válida de la evolución de las variables biológicas y psicológicas más significativas para dar una visión integral del proceso de la adolescencia. Nos hemos propuesto investigar los cambios somáticos, niveles hormonales $y$ dimensiones psicológicas relevantes en el adolescente chileno de distintos niveles socioeconómicos. La presente comuricación es parte de la investigación anteriormente señalada, y se refiere a algunos parámetros antropométricos encontrados en adoles. centes chilenos, de sexo masculino, de nivel socioeconómico medio-alto.

\section{MATERIAL Y METODO}

La muestra utilizada en la presente investigación es dè tipo intescionada. Se tomaron sujetos de nivel 
socioeconómico medio-alto, detinidos según dos de los criterios de Barylary y Oxley ${ }^{1}$ : nivel de escolaridad y ocupación del padre. Estos indicadores son los que mejor discriminan entre los niveles socioeconómicos. Así los individuos estudiados eran hijos de medianos y grandes empresarios, empleados directivos que hubieran completado su educación secundaria y/o profesionales universitarios. La muestra defunitiva quedó constituida por 263 individuos pertenecientes a un colegio privado desde quinto básico a cuarto medio. Los sujetos incluidos en la investigación fueron aquellos que cumplian años en el período comprendido en tre Marzo y Mayo, se ofrecieron voluntariamente $y$ contaban con la debida autorización de sus padres. La restricción respecto al período de edad en' que el sujeto cumplía años obedece al fin de no tenet gran dispersión de las edades, criterio fundamental cuando se van a estudiar parámetros de crecimiento en una muestra relativamente pequeña. La metódica para el estudio antropométrico usada fue la descrita por Tanner $y$ col. 2 . Se estudiaron los siguientes parámetros: peso, talla, talla sentada, envergadura, diámetro biacromial, diámetro bitracantéreo, longitud de muslo, circunferencia de muslo, longitud de pierna, circunferencia de pierna, longitud de brazo, circunferencia de brazo, longitud de antebrazo, circunferencia de antebrazo. longitud de mano, palma, dedo medio mano, pie, planta y dedo medio del pie.

Los diámetros biacromial y bitrocantéreos se midieron usando un compás obstétrico y haciendo la conversión a centímettos después de rèalizada la medida.

Las mediciones de los restantes segmentos fueron hechas con huincha flexible empleando los siguientes puntos de referencia para cada segmento del lado derecho: muslo (distancia entre el punto medio del pliegue inguinal y el punto medio de ta rótula del mismo lado), circunferencia de muslo (en la intersección del $1 / 3$

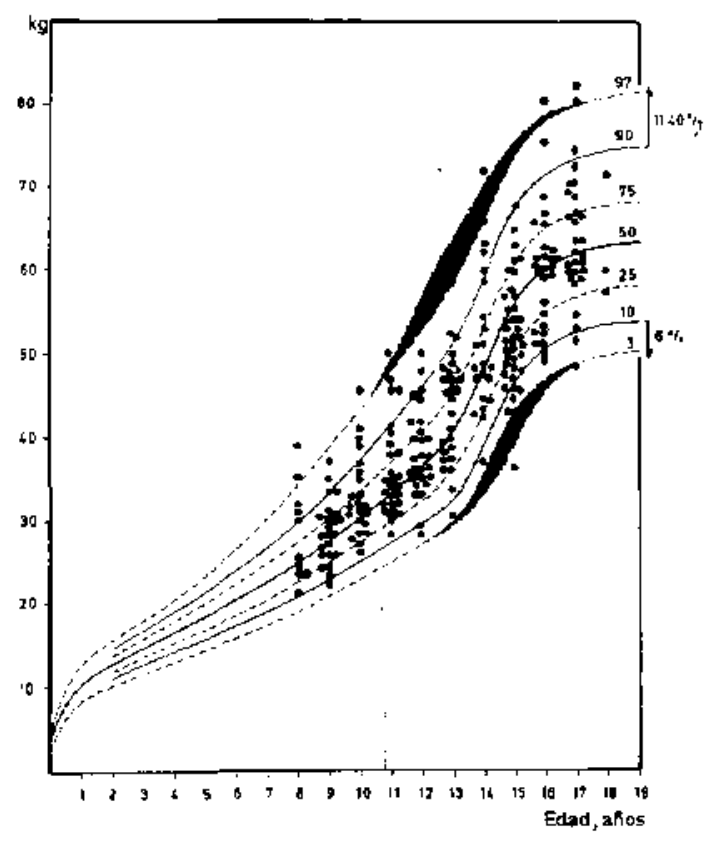

superior con los 2/3 inferiores), pierna (distancia comprendida entre el punto medio de la rótula proyectado hacia el borde interno de la tibin y el maléolo distal interno de la tibia), circunferencia de pierna (en el punto medio de la pierna), brazo (trayecto desde la prominencia del acromion, hasta el borde inferior del oléctanon, con codo en flexión de $90^{\circ}$ ), circunferencia de brazo (en el punto medio del brazo), antebrazo (distancia entre el borde inferjor de otécranon $y$ la apófisis estiloides en el borde del cúbito), circunferencia antebrazo (en el punto medio de antebrazo), mano (distancia comprendida entre el pliegue de la nuñeca y el extremo distal del dedo medio de la mano), palma (distancia entre el pliegue de la muñeca y el pliegue metacarpo-falángico de flexión del dedo medio, en extensión), dedo medio (distancia entre el pliegue metacarpofalángico del dedo medio y su extremo distal), pie (medida comprendida entre el borde externo del talón $y$ el extremo distal del tercer ortejo), planta (trecho comprendido entre el borde externo del talón hasta pliegue metatarso-falángico de flexión del tercer ortejo).

\section{RESULTADOS}

En la Fig. 1 a puede observarse el peso de los individuos estudiados dibujado sobre una curva de crecimiento de Tanner ${ }^{3}$. Sólo $11,4 \%$ de los individuos se encuentran por sobre el P 90 y el $6 \%$ bajo el P 10 ; de manera que el $83 \%$ de la muestra estudiada se ubica entre el P 90 y P 10 de la curva normal de la población inglesa descrita por Tantner. En la Fig. I b se muestra las tallas de los adolescentes estudiados, dibujados sobre la

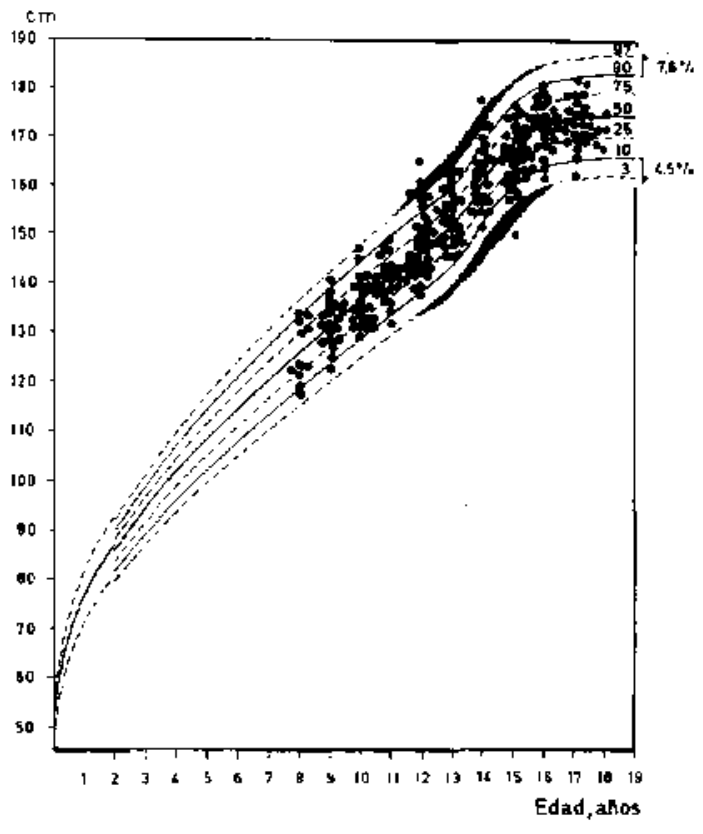

b

Fig. 1. a) Valores individuales del peso de adolescentes, varones, chilenos, de condición socioeconómica media alta, dibujados sobre una curva normal de adolescentes ingleses según Tanner. b) Valores individuales de la talla de adolescentes, varones, chilenos de condición socioeconómica media alta, dibujados sobre una curva normal de adolescentes ingleses según Tanner. 
misma curva de Tanner. Sólo $4,5 \%$ de los indivi. duos estudiados presentan talla inferior al $P 10$ y el $7,6 \%$ por sobre el P 90 ; es decir el $87,9 \%$ de la muestra constituida por adolescentes chilenos se encuentra entre el P 90 y $\mathbf{P} 10$ de las tablas de crecimiento construida por Tanner. En la Fig. 2 se muestra una curva de velocidad de crecimiento de los adolescentes ingleses; sobre ella se ha representado mediante un punto el valor promedio del incremento para cada edad de los adolescentes chilenos estudiados. Puede observarse que todos estos puntos caen dentro de los P 90 y 10 descritos por Tanner y el período máximo de crecimiento se observa aproximadamente a los 14 años con un crecimiento promedio de $\pm 10 \mathrm{~cm}$ entre los 13 y 14 años.

En las Tablas 1 a 4 pueden observatse los valores promedios de talla sentada, envergadura, - diámetro biacromial, diámetro bitrocantéreo, longitud de muslo, circunferencia de muslo, longitud de pierna, circunferencia de pierna, longitud de brazo, circunferencia de brazo, longitud de antebrazo, circunferencia de antebrazo, mano, palma de la mano, dedo medio de la mano, pie y dedo medio del pie de la población de adolescentes estudiados en el presente estudio.

\section{COMENTARIO}

De acuerdo con los resultados obtenidos en la

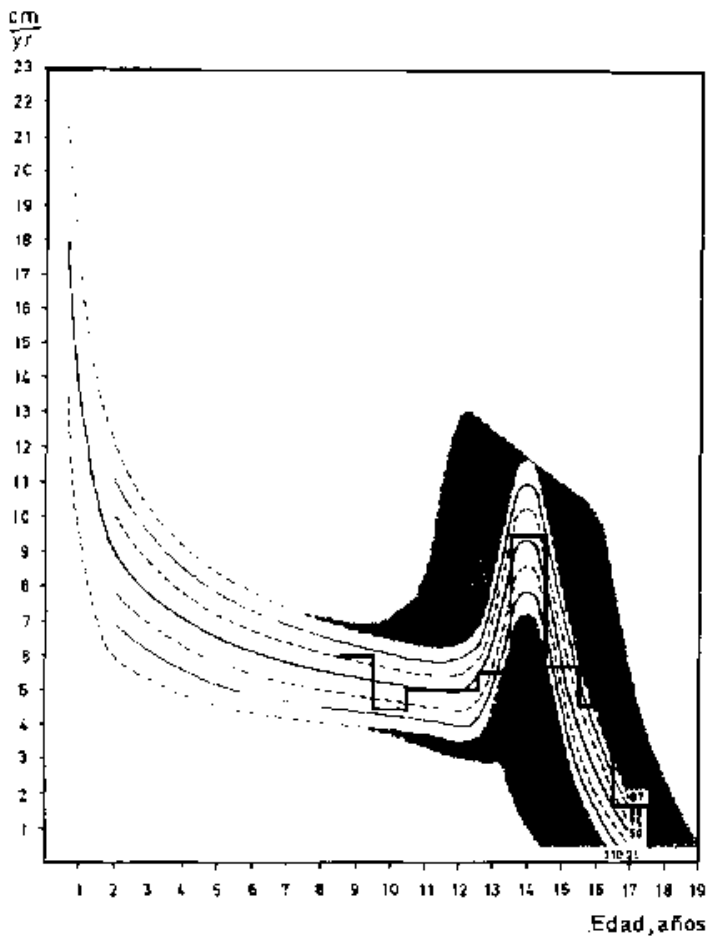

Fig. 2. Sobre una curva de velocidad de crecimiento correspondjente a adolescentes ingleses se ha representado, mediante un punto el valor promedio del incremento para cada edad de los adolescentes chilenos.

presente investigación la talla, peso y ritmo de crecimiento de adolescentes chilenos de nive]

Tabla 1.

Antropometria en varones adolescentes

\begin{tabular}{|c|c|c|c|c|c|c|c|c|c|c|c|c|c|c|c|c|c|c|}
\hline \multirow[t]{2}{*}{$\begin{array}{l}\text { ldad } \\
\text { (ữos) }\end{array}$} & \multicolumn{3}{|c|}{$P_{e x}\left(K_{g}\right)$} & \multicolumn{3}{|c|}{ Altova $(\mathrm{cm})$} & \multicolumn{3}{|c|}{ 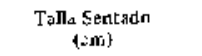 } & \multicolumn{3}{|c|}{$\begin{array}{c}\text { Diamelso } \\
\text { linvergadura }(\mathrm{cm})\end{array}$} & \multicolumn{3}{|c|}{$\begin{array}{l}\text { Dixmet to } \\
\text { Biacromial (Lm) }\end{array}$} & \multicolumn{3}{|c|}{ Bilrocanterco $(x \mid n)$} \\
\hline & $x$ & D.1. & $\pi$ & $x$ & D.t. & $\pi$ & $x$ & D. I: & $n$ & $\bar{x}$ & D.E. & n & $\overline{\mathrm{x}}$ & D.l: & $n$ & $\bar{x}$ & $1 \mathrm{~L}$. & $n$ \\
\hline 8 & 28.82 & 5.85 & $(! 1)$ & $127,0,3$ & 7,35 & (11) & 68.18 & $3,5 ?$ & 411। & 127,09 & 6,40 & (I1) & 26,25 & 3.44 & $(9)$ & 23,04 & 3,21 & (9) \\
\hline 9 & 29.41 & 2.95 & (23) & 133.60 & 4,74 & 120) & 69,55 & 3,02 & $120)$ & 132,73 & 5.30 & (19) & 28,12 & 2,01 & $12 \geqslant 1$ & 22,28 & $1, \geq 3$ & {$[20)$} \\
\hline 10 & 33.15 & 5,跑 & (21) & 137.75 & $6.1 ?$ & (34) & $22 . \infty$ & $2.9+$ & $122)$ & 138,66 & 5.98 & (21) & 29,30 & $1,1,66$ & $(22)$ & 22.88 & 1,28 & (22) \\
\hline 11 & 35.92 & 5.12 & !26) & 142,24 & 6,64 & $(26)$ & 73,56 & 3,06 & $126 !$ & 143,73 & 1.09 & (21) & 30.86 & 2,21 & (24) & 23,87 & 1,35 & $(25)$ \\
\hline 12 & 34.96 & 5,87 & $(28)$ & 147.86 & 7,40 & $(27)$ & 74,76 & 4,35 & $128 j$ & $14 B_{7} 86$ & 7,53 & $130 \mathrm{j}$ & 31.17 & 2,28 & (2T) & 23,75 & 2,12 & $\{27 ;$ \\
\hline 13 & 45.61 & 3.73 & $i 28$ & 153,70 & $6, \overline{7}]$ & $\{32\}$ & 77,58 & 3,44 & $91 j$ & 154,92 & 9,72 & (33) & 33,19 & 2,22 & $(30)$ & 26,12 & 1,77 & {$[30]$} \\
\hline 14 & 52.84 & 8,68 & (25) & 163.16 & 9.06 & (25) & 83,34 & $4,6 \mathrm{~A}$ & $\{22\}$ & 166.48 & 9.21 & $\{25\}$ & 34.06 & 3,25 & $\{2 \mid\}$ & 26,54 & 2,52 & (23) \\
\hline 15 & 56.26 & 6,98 & (37) & 168.67 & 0,63 & (38) & 85.94 & 3.79 & (38) & 167.30 & 7.62 & (33) & 35,42 & 2,48 & ¿38ł & 27,58 & 1.90 & (34) \\
\hline 16 & 61.39 & 3,45 & $(25 i$ & 17315 & 5,69 & (24) & 37,65 & 3,96 & (26) & 174.32 & 6,70 & (2B) & 39,28 & 4,25 & (22) & 28,17 & 2.52 & (26) \\
\hline 17 & $6 \pm .25$ & 8,22 & (24) & 174.83 & 6,36 & (26) & 90.02 & 3,54 & (23) & 176.95 & $7,9 ?$ & $(25 i$ & 28,20 & 2.09 & $121 !$ & 29.32 & 1,02 & (24) \\
\hline 18 & 64,06 & 5,83 & ( 5$)$ & & & & & & & & & & & & & & & \\
\hline
\end{tabular}

Tabla 2.

Antropometría en varones adolescentes

\begin{tabular}{|c|c|c|c|c|c|c|c|c|c|c|c|c|c|c|c|c|c|c|}
\hline \multirow[t]{2}{*}{$\begin{array}{l}\text { t.dad } \\
\text { (años) }\end{array}$} & \multicolumn{3}{|c|}{$\begin{array}{c}\text { Musis } \\
\text { L.ongilud iall) }\end{array}$} & \multicolumn{3}{|c|}{$\begin{array}{l}\text { Musk Circun- } \\
\text { Ferencin timl }\end{array}$} & \multicolumn{3}{|c|}{$\begin{array}{c}\text { Merna } \\
\text { L.ongltid (cun) }\end{array}$} & \multicolumn{3}{|c|}{ 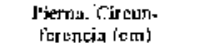 } & \multicolumn{3}{|c|}{$\begin{array}{c}\text { Hrazo } \\
\text { Longitud }(\mathrm{cm})\end{array}$} & \multicolumn{3}{|c|}{$\begin{array}{l}\text { Hrazo. Circun- } \\
\text { Eurencid (sm) }\end{array}$} \\
\hline & $\vec{x}$ & D.1: & $\mathbf{n}$ & $\dot{x}$ & 1).1:. & ח & $x$ & บ.I:.- & נו & $x$ & D.E. & n & $x$ & D.I: & $n$ & $\bar{x}$ & D.t.. & $n$ \\
\hline 8 & 28,14 & 2,06 & (II) & 36.77 & 5.76 & (1I) & 31,68 & 3,54 & $(11)$ & 25,32 & 2,72 & (I1) & 26,18 & 2,19 & (11) & 20,86 & 2,54 & (I1) \\
\hline 9 & 29,93 & 2.49 & (20) & 36,26 & $2.2 ?$ & (19) & 32.69 & 2,73 & (21) & 24,78 & 2,62 & (21) & 25.69 & 3,63 & $\{21\}$ & $20,0 ?$ & 1,27 & (21) \\
\hline 10 & 31.43 & 1.76 & $\{2\}$ & 36.22 & 3,94 & (22) & 35.07 & 2,29 & 122) & 26,23 & 2,12 & (21) & 26,54 & 1,69 & $\{21\}$ & 21,20 & 2.11 & (22) \\
\hline 11 & 32,89 & 3,31 & (24) & 38,30 & 3.16 & (25) & 36,34 & 2,53 & (25) & 27.16 & 2.44 & (24) & 28,27 & 1,36 & $\{26\}$ & 21,12 & 1.79 & (25) \\
\hline 12 & 33.90 & 2,47 & (28) & 39.18 & 3,33 & (27) & 37.05 & $3.0 ?$ & (27) & 27.68 & 3,20 & $(26)$ & $2 B, 36$ & 1,67 & (27) & 22,31 & 2,22 & $(27)$ \\
\hline 13 & 35.98 & 2.11 & (32) & 41.40 & 3,13 & \{31\} & 38,93 & $9.4 ?$ & $131\}$ & 29.32 & 2,57 & (31) & 30.50 & 1,90 & (31) & 22,96 & 2,33 & i33! \\
\hline 14 & 38.38 & 2.81 & (25) & 44.06 & 3.37 & (24) & 41,85 & 2.75 & $24 ;$ & 31,80 & 2.70 & (23) & 31,61 & 2,32 & (22) & 24,56 & 2,76 & (23) \\
\hline is & $39,6 ?$ & 2,59 & (3s) & 43,86 & 3.72 & [25, & 42,22 & 3.28 & (34) & 29.67 & 3.08 & (32) & 32,93 & 2.70 & (38) & 24.15 & 2.08 & (3?) \\
\hline 16 & 40,82 & 2,59 & $\{25\}$ & 46.82 & 4,34 & (2Si & 43.59 & 2,22 & (21) & 32,20 & 3,09 & (25) & 34,70 & 2,86 & (25) & 26,24 & 2.35 & (23) \\
\hline 17 & 42,16 & 2,64 & $(25)$ & 48.33 & 4.07 & (24) & 43.52 & 3,14 & (23) & 33.024 & 3.73 & (24) & 34,20 & 2.26 & (22) & 27,22 & 2,07 & (25) \\
\hline
\end{tabular}


Tabla 3.

Antropometría en varones adolescentes

\begin{tabular}{|c|c|c|c|c|c|c|c|c|c|c|c|c|c|c|c|}
\hline \multirow[t]{2}{*}{$\begin{array}{l}\text { Edad } \\
\text { (años) }\end{array}$} & \multicolumn{3}{|c|}{$\begin{array}{c}\text { Antebrazo } \\
\text { Longitud (cm) }\end{array}$} & \multicolumn{3}{|c|}{$\begin{array}{l}\text { Antebrazo. } \\
\text { Circunferencia (cm) }\end{array}$} & \multicolumn{3}{|c|}{$\begin{array}{c}\text { Mano } \\
(\mathrm{cm})\end{array}$} & \multicolumn{3}{|c|}{$\begin{array}{l}\text { Palma } \\
(\mathrm{cm})\end{array}$} & \multicolumn{3}{|c|}{$\begin{array}{l}\text { Dedo Medio } \\
\text { Mano }(\mathrm{cm})\end{array}$} \\
\hline & $\bar{x}$ & D.E. & $\mathrm{n}$ & $\bar{x}$ & D.E. & $\mathrm{n}$ & $\bar{x}$ & D.E. & $\mathrm{n}$ & $\overline{\mathrm{x}}$ & D.F. & n & $\overline{\mathrm{x}}$ & D.L. & $\pi$ \\
\hline 8 & 19,68 & 1,79 & (11) & 17,36 & 2,02 & (11) & 14,34 & 1.05 & (11) & 8,23 & 0.75 & (11) & 6,24 & 0,79 & (I1) \\
\hline 9 & 20,17 & 1,36 & (21) & 17,07 & 1,52 & (21) & $1 \$, 08$ & 1,03 & (1?) & 8.57 & 0,79 & $\{21\}$ & 6,58 & 0,56 & (19) \\
\hline 10 & 20,64 & 1,55 & (22) & 17,11 & 1,66 & (22) & 15,00 & 0,77 & (21) & 8,55 & 0,50 & (21) & 6,47 & 0,47 & (20) \\
\hline 11 & 21,81 & 1,22 & (26) & 17,84 & 1,66 & (25) & 15,94 & 0,85 & (24) & 9,08 & 0,50 & (24) & 6,94 & 0,71 & (26) \\
\hline 12 & 22,82 & 1,19 & $(2 B)$ & 18,31 & 1,73 & (26) & 16,36 & 0,90 & (28) & 9,55 & 0,77 & (29) & 6,83 & 0,50 & (27) \\
\hline 13 & 24,00 & $t$, so & (32) & 19,14 & 1.79 & (31) & 17,18 & 0,93 & (30) & 9,90 & 0.59 & (30) & 7,30 & 0,66 & (21) \\
\hline 14 & 25,81 & 2,32 & $(24)$ & 20,00 & 1,90 & (24) & {$[8,08$} & 1,20 & $(25)$ & 10,29 & 0.83 & (24) & 7,70 & 0,62 & (20) \\
\hline 15 & 25,56 & 1,61 & (38) & 19.72 & 2,19 & (37) & 18,41 & 1,23 & (38) & 10,51 & 0.77 & (36) & 7,96 & 0,65 & (36) \\
\hline 16 & 27,31 & 2,39 & (26) & 21,37 & 1,43 & (24) & 18,82 & 1,13 & $(25)$ & 10,72 & 0.60 & (25) & 8,26 & 0,74 & (23) \\
\hline 17 & 27.13 & 1,39 & (27) & 22,31 & 1.64 & (27) & 19,10 & 1,27 & (24) & 10,89 & 0,85 & (53) & 8.26 & 0,72 & (25) \\
\hline
\end{tabular}

Tabla 4

Antropometsía en varones adolescentes

\begin{tabular}{|c|c|c|c|c|c|c|c|c|c|}
\hline \multirow[t]{2}{*}{$\begin{array}{l}\text { Edad } \\
\text { (años) }\end{array}$} & \multicolumn{3}{|c|}{ Pie (cm) } & \multicolumn{3}{|c|}{$\begin{array}{c}\text { Planta } \\
\text { Pie (cm) }\end{array}$} & \multicolumn{3}{|c|}{$\begin{array}{c}\text { Dedo Medio } \\
\text { Pie }(\mathrm{cm})\end{array}$} \\
\hline & $\dot{\mathrm{x}}$ & D.E. & $\pi$ & $\bar{x}$ & D.E. & n & $\bar{x}$ & D.E. & n \\
\hline 8 & 18,22 & 1,71 & (11) & 16,09 & 1,43 & (I1) & 2,03 & 0,55 & (11) \\
\hline 9 & 18,93 & 1,43 & (21) & 16,90 & 1,06 & (2I) & 2,10 & 0,61 & (20) \\
\hline 10 & 20,31 & 1,31 & (2l) & 17,75 & 1,19 & (21) & 2,54 & 0,49 & (21) \\
\hline 11 & 21,31 & 1,56 & (26) & 18,33 & 1,41 & (25) & 2,98 & 0.57 & (26) \\
\hline 12 & 22,07 & 1,55 & (27) & 19,22 & 1,34 & (27). & 2,87 & 0,53 & (27) \\
\hline 13 & 23,20 & 1,92 & (32) & 19,97 & 1,57 & (31) & 3,12 & 0,60 & (28) \\
\hline 14 & 23,98 & 1,43 & (23) & 21,00 & 1,36 & (23) & 3,19 & 0,62 & (22) \\
\hline 15 & 23,70 & 1,59 & (38) & 20,62 & $1, \$ 2$ & (38) & 3,03 & 0,57 & (36) \\
\hline 16 & 24,67 & 1,60 & (26) & 21,35 & 1,32 & (26) & 3,25 & 0,58 & (24) \\
\hline 17 & 24,48 & 1,86 & (27) & 21.59 & 2,07 & (27) & 3,13 & 0,52 & (23) \\
\hline
\end{tabular}

socioeconómico medio-alto es similar a adoles. centes británicos. Ha sido previamente descrito que el pico de velocidad máxima de crecimiento varía de un niño a otro. Marshall y Tanner encontraron un valor prome dio de $10,3 \mathrm{~cm} x$ año con una DE de $\pm 1,54 \mathrm{~cm} x$ año en 49 niños sanos que fueron medidos cada 3 meses por un sólo investigador ${ }^{3}$. Los adolescentes de esta muestra presentaron un mayor crecimiento aproximado de $10 \mathrm{~cm}$ entre $\operatorname{los} 13$ y 14 años. Así aproximadamente en el año del mayor crecimiento los niños crecen el doble de $\mathrm{cm}(10 \mathrm{~cm})$, que crecían anualmente antes del estirón $(5 \mathrm{~cm})$.

Cuando se compara el comportamiento del crecimiento de adolescentes chilenos de condición socioeconómica medio-aita, como el de esta muestra, con el de adolescentes chilenos de condición socioeconómica baja, la diferencia es ostensible. Esto puede observarse en la Fig. 3 donde, los resultados obtenidos en esta investigación, se comparan con los publicados por Avendaño 4, estudio efectuado en estratos socioeconómicos deficientes. Estos resultados son concordantes con los comunicados por de la Fuente y $\operatorname{col}^{5}$ quienes encontracon que adolescentes chilenos de nivel socioeconómico alto

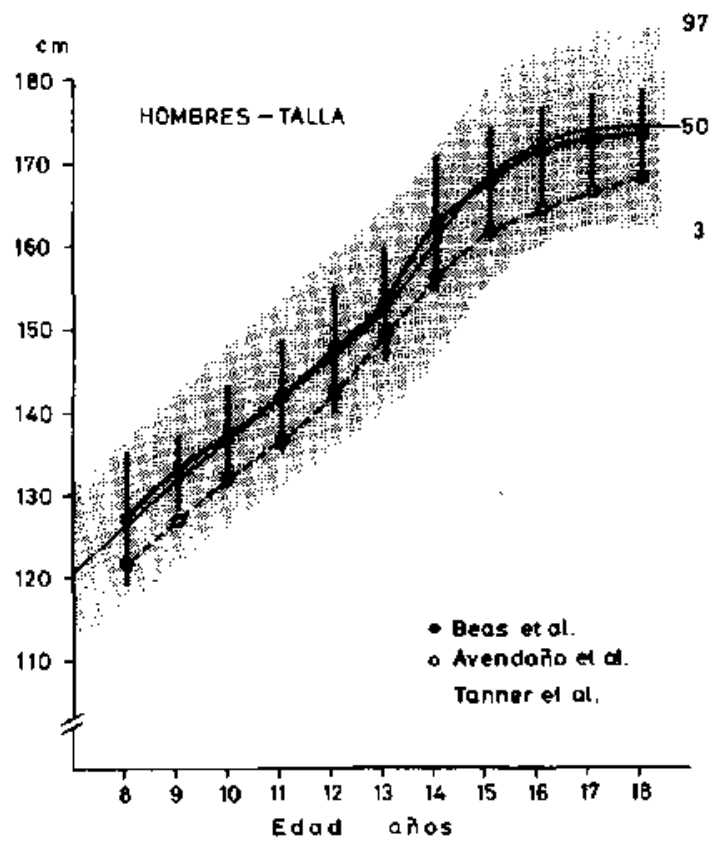

Fig. 3. Curva de crecimrento de adolescentes ingleses y chilenos de clase socioeconómica media alta $(\bullet$ y baja (o). 
cursan por el $\mathrm{P} 50$ de los patrones de nommalidad propuestos por el NCHS ${ }^{6}$ (USA, 1976) ubicándose estos niños, entre +1 y +2 DE sobre las curvas publicadas por Avendaño, de adolescentes provenientes del Area Norte de Santiago. Esta misma situación ha sido confirmada por Muzzo, en ninias adolescentes chilenas provenientes de estratos socioeconómicos bajos 7 del Area Sur de Santiago. Es bien sabido que el crecimiento de un individuo es la resultante de un fenómeno biológico nultifactoriale. Entre estos factores destacan los genéticos y los ambientales (nutrición, salud, condiciones socioculturales, etc.). Así el potencial genético de un individuo se expresa. en mayor o menor cuantía, si el medio que le rodea es propicio para aportarle las condiciones necesarias para un desarrollo y crecimiento normal. Al analizar las talias comprometidas de adolescentes chilenos de bajas condiciones socioeconómicas, la pregunta obvia es, cuánto de este compromiso es de causa genética y en qué magnitud lo es del factor ambiental.

Valenzucla $a^{9}$ ha estudiado las relaciones que existen entre estrato socioeconómico, porcentaje de mezcla aborígen y origenes de los apellidos en diferentes poblaciones de Santiago y Temuco, comprobando que el porcentaje de mezcla aborigen es casi inexistente en los estratos socioeconómicos altos y mayor en los estratos bajos y se inclina a pensar que los factores genéticos podrian explicar parte de las diferencias de estatura en los diferentes niveles socioeconómicos. Sin embargo, grupos de individuos de deficientes condiciones socioeconomicas, se expresan con crecimiento similar al de los normales cuando se mejora su condición, a pesar de poseer diferentes "componentes genéticos"10,11. Habicht" 12 ha demostrado, basado en diferentes estudios, que hasta los 6 meses de edad nifios provenientes de diferentes grupos étnicos crecen uniformemente en talla y peso. Después de los 6 meses de edad, tanto los niños provenientes de países desarrollados, como los de clase media-alta de comunidad en vías de desatrollo, crecen por canales muy similares. Sólo aquellos de condición socioeconómica baja se apartan de estos canales. Así por ejemplo, los japoneses emigrados a los Estados Unidos presentan igual talla que el norteamericano promedio, en tanto que este promedio en Japón en esa época estaba por debajo del promedio de $\mathrm{EU}^{13}$. En nuestro medio, de la Fuente, en un estudio antropométrico y nutricional en escolares de nivel socioeconómico alto concluye que "el factor socioeconómico ambiental prima sobre el genética logránd ose pesos y tallas relativamente uniformes cuando el nivel socioeconómico es muy elevado ${ }^{14}$. Atalah ha demostrado que el estado nutricional de los adolescentes provenien- tes del Area de Salud Norte de Santiago es deficiente ${ }^{15}$. Es probable, que en el eștudio comparativo que hemos hecho en la Fig. 4, los adolescentes del Area Norte, con un adecuado aporte nutritivo, expresarian una talla similar a la de aquellos provenientes de condiciones socioeconómicas aceptables, chilenos o británicos. Es posible que, corregidos los factores ambientales, el dimorfismo genético se exprese dentro de las variaciones del percentil 10 y 90 , que nos hemos prefijado como patrones "normales" de crecimiento.

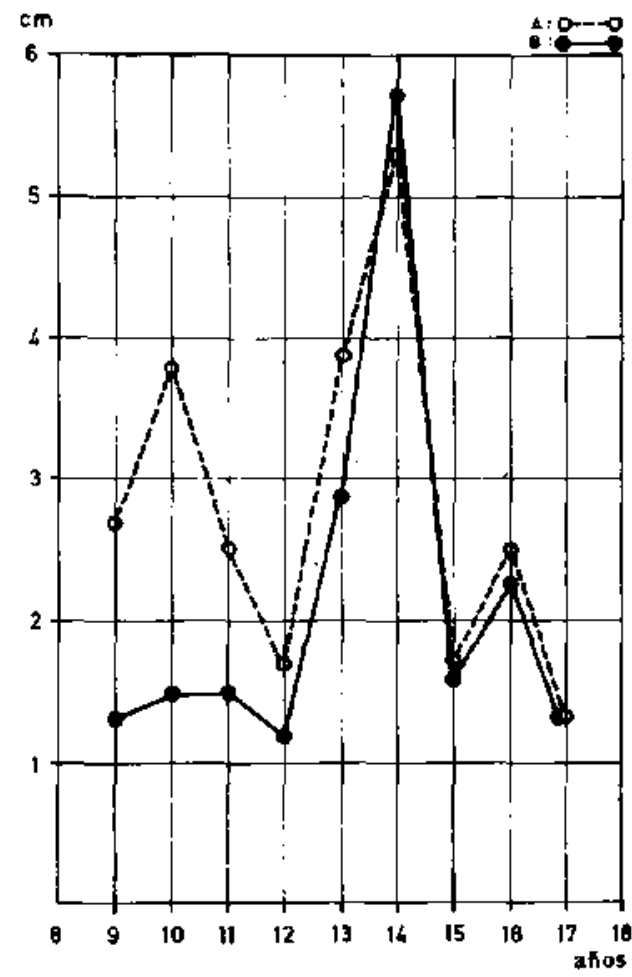

Fig. 4. Crecimiento del tronco o- 0 y extremidades $\longrightarrow$ de adolescentes chilenos.

Por las razones expuestas anteriomente, hemos optado por el uso de las curvas de Tanner. Ellas han sido construidas a través del seguimiento longitudinal de un número importante de individuos, con adecuados instrumentos estadísticos. Presentan la ventaja de no ses sólo curvas clásicas de crecimiento y desarrollo sino que además curvas de "velocidad de crecimiento", pronóstico de talla, etc. Además están expresadas en "décimas" de años, lo que facilita significativamente el tratamiento de la información obte. nida con computadoras.

En relación a los otros segmentos corporales tambiér es bien conocido que se acelera su crecimiento durante la adolescencia. Sin embargo, este incremento no es uniforme. Falkner y 
Tanner ${ }^{16}$ han descrito que durante ef mayor crecimiento, crece más, en longitud, el tronco que las piernas. En el presente estudio no obseryamos esa diferencia. La Fig. 5 muestra que tanto el tronco como las extremidades, crecieron en total, en forma similar. Sin embargo, en la misma figura, puede observarse que el crecimiento del tronco se produjo fundamentalmente alrededor de los 14 años, coincidiendo con el período del estirôn general del organismo, y probablemente los mecanismos fisiológicos homonales que condicionan ambos fenómenos sean los mismos. Por el contrario, el crecimiento total de las extremidades, se produjo por la sumatoria de dos picos de crecimiento: uno alrededor de los 10 años y el segundo alrededor de los 14 años; se podria elucubrar, que el primer pico de creci. miento, podría estar condicionado por la secreción de los andrógenos suprarrenales, especialmente dehidroepiandrosterona, y que el segundo, se produciría por mecanismos fisiológicos similares al del estirón común de todo el organismo.

Además se ha descrito ${ }^{16}$ que el fenómeno del estirón no se produce simultáneamente en todos los segmentos del cuerpo. Esta diferencia en velocidad y tiempo en que se produce el crecimiento pareciera recordar la gradiente de maduración del crecimiento "in útero": al nacer, el tamaño del cráneo está mucho más cercano al tamaño del adulto, que el del tórax o las extremidades. Por otra parte con las extremidades existe una gradiente de crecimiento inversa: las manos y los piés crecen antes que las piernas y los antebrazos, y éstos a su vez, maduran antes que los brazos y los muslos. Así, en un momento determinado del estirón, el adolescente aparece con manos y piés desproporcionadamente grandes. Probablenente el crecimiento de las extremidades adquiere su máximo pico de velocidad antes que el tronco, y los piés son los primeros en crecer, con la posible excepción de la cabeza' ${ }^{16}$. Del estudio presente no es posible sacar similares conclusiones al respecto por su diseño transversal, en diferentes edades, y no longitudinal. Sin embargo, del análisis de los diferentes segmentos corporales, durante el período de la adolescencia, nos ha parecido importante, con el fin de contar, en forma empírica, con los valores promedios y las DE de nuestra población, para poder realizar un diagnóstico con precisión, cuando ellos se alejan de la normalidad. Es bien sabido que existen numerosas afecciones endocrinas o geneticas, que afectan en forma característica y muy particular algunos de estos segmentos corporales.

\section{AGRADECIMIENTOS}

Este trabajo fue financiado en parte por la Dirección General de Deportes (DIGEDER).

Nuestros agradecimientos especiales a Eriks Himmel y Beatriz Zegers por su valiosa colaboración en el disejo de la investigación y sugerencias para el estudjo estadistico y presentación de los resultados.

\section{REFERENCLAS}

1. Barviry, R. y' Oxley, J.: Documento Clínica Psiquiátrica Universitaria, Año 1960.

2. Tanner, J.M.: Growth at Adolescence, 2nd ed. Blackwell Scientific Publications, Oxford, 1962.

3. Marshall, W.A. and Tanner, J.M.: Variation in the pattern of pubertadl changes in boys. Arch. Dis. Child. 45: $13,1970$.

4. Avendaño y coli: Antropometría de escolares chilenos del Area Norte de Santiago. Cuadernos Médico-Sociales. 12: 5, 1975 .

5. De ta Fuente, M. Margozzini, J., Maiva, M.E Yentzen, G. y Prodo, M.: Antropometría de.Escola res de Nivel Socio Económico Alto. Estudio Descriptivo. Rev. Chil. Pediatr. 54. 353, 1983.

6. Nationai Center for Health Statistics: Health Examination Survey Data. Vital Statistics Report. 25 . No 3. Supplement June 1976.

7. Muzzo, S., Burrow, A., García, H. y Gonzalez, T.: Retraso estatural en adolescente de clase mediabaja. Rev. Chil. Pediatr. 54: 24, 1983.

8. Beas, $F_{\text {. }}$ : Endocrinología del crecimiento y desarrollo. En Pediatría II, Meneghelo, J. y col. Ed. Mediterráneo, Santiago, Chile, pp. 1174-1179, 1985.

9. Valenzuela, $C$.: Marco de referencia sociogenético para los estudios de Salud Pública en Chile. Rev. Chil. Pediatr. 55: 123, 1984.

10. Donoso, G., Undurraga, O., Weschler, 1 , Bauza, I. y Saez, $M_{\text {.: }}$ Estudio del crecimiento pondoestatural del niño de 0 a 2 años. Pediatría, Santiago, Chile. 5 : 135,1962 .

11. Donoso, G. y Monckeberg, F.: Desnutrición Infantil, I. Consideraciones generales y epidemiología. Rev. Chil. Pediatr. 36: 301, 1965.

12. Habicht, J.P., Mortorell, R., Yarbrough, C., Molina. R.M. and Klein, R.E.: Height and weight standards for preschool children. How relevant are ethnic differences in growth potential. lancet 1: 61l, 1974.

13. Greulich, W: A comparison of the physical growth and development of American born and native japaneese children. Ant. J. Phys. Antropol. 15: 489, 195 ?

14. De lo Fuente. M., Margozzini, J., Maira, ME., Yentzen, G. y Prado Margarita: Antropometria y Estado Nutritivo de Escolares de. Nivel Socioeconómico Alto según origen de los apellidos. Rev. Chil. Pediatr. 359, 1983.

15. Atolah, E. y col:: Evaluación Nutricional de una población infanto-juvenil del Area Norte de Santiago. Pediatria. 22: 227, 1979.

16. Folkner, F. and Tanner, J.M.: "Human Growth. Post-Natal Growth. Plenum Publ. N.Y. \& Lonđon, 1978. Yo1. II. pp. 142-145. 